\title{
Die Stadt als „Werkzeug“ - ein neuer Ansatz für die Stadtforschung?
}

\author{
Rezension zu Uwe Prell (2020): Die Stadt. Eine Einführung für die \\ Sozialwissenschaften. Opladen/Toronto: Barbara Budrich
}

\section{Alexander Krahmer}

Abb. 1 Titel des Buches (Quelle: Verlag Barbara Budrich)

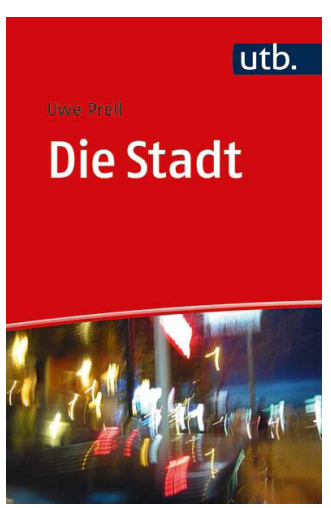

\section{Einleitung}

Das Buch Die Stadt des Politikwissenschaftlers, Historikers und freien Autors Uwe Prell möchte nicht nur in die Stadtforschung einführen, sondern dieser gleichzeitig einen neuen Ansatz vorschlagen. Entsprechend präsentiert der Band nicht nur Fragen der Stadtforschung, ihre Disziplinen und einige Theorien, sondern prüft diese zugleich auf Verständnis und Wissen von „der Stadt“.

Zunächst führt das erste Kapitel in die „Mühen der interdisziplinären Forschung“ ein. Prell diagnostiziert als zentrales Problem, dass ein übergreifender Stadtbegriff fehle. Das zweite Kapitel schlägt dann vor, die Stadt als „Werkzeug“ zu verstehen, ließe diese sich doch auf verschiedene Weise „nutzen“ und steigere, gleich einem Werkzeug, unsere Möglichkeiten. Das dritte Kapitel wirbt für einen „holistischen Ansatz“, wozu es durch zahlreiche Disziplinen (u. a. Soziologie, Philosophie, Geographie, Ökonomie sowie Rechts- und Politikwissenschaft) und Theorieansätze führt (u. a. von Aristoteles, Max Weber, Georg Simmel, der Chicago School und Saskia Sassens Global-Cities-Ansatz), um schließlich aus zwölf Sprachen einen Allgemeinbegriff „der Stadt“ zu entwickeln. Darauf aufbauend führt das vierte Kapitel in „Stadtkonzepte“ und „Stadtthemen“ ein, während das fünfte Kapitel die wichtigsten Ergebnisse zusammenfasst. Selbst wenn der Band durchaus interessante Anregungen (und auch Exkurse) bietet, können sein holistischer Ansatz und der entwickelte Stadtbegriff nicht wirklich überzeugen. Warum das so ist, möchte ich im Folgenden zeigen.

\section{Die Stadt - ein ,Werkzeug“?}

Bereits im „Berlin Handbuch“ (1992 gemeinsam mit Horst Ulrich herausgegeben) und in seiner 2005 erschienenen Dissertation Berlin - Bühne des Wandels. Ein Reisebericht (Prell 2005) beschäftigte sich der Autor mit Städten. Basis für sein neues Buch Die Stadt sind Ergebnisse seiner Habilitation (Prell 2016), in der er den Versuch unternahm, das Stadtwissen 
auf seine „Essenz“ zu prüfen und in einem holistischen Ansatz zu vereinen (Prell 2016: 18 ff., 213). Die Stadt hält daran fest, selbst wenn „,[z]ahlreiche Kolleginnen und Kollegen“ diesen Weg ablehnen (Prell 2020: 9) - zu Recht, wie man hier zu sagen geneigt ist. Denn selbst wenn dievielen Einzeldisziplinen und das Fehlen eines Stadtbegriffs mitunter Schwierigkeiten bereiten, scheint es doch ratsam, dem Wunsch nach einem „Gesamtbild“ (137) nicht einfach die Komplexität des Gegenstands und die Multiperspektivität auf ihn unterzuordnen. Insofern ist Prells Wunsch - oder „Obsession“ (Prell 2016: 273) - nach „holistischen Antworten“ durchaus anfechtbar (vgl. Saunders 1987; Häußermann/Siebel 2004: 89 ff.). Er selbst kritisiert die Stadtforschung dafür, sich nur „,in die Breite“ zu entwickeln und kaum „Fortschritt“zu machen (Prell 2020: 9). Darin, eine „alle Fächer übergreifende Definition“ (37) zu liefern, sieht er seinen eigenen Auftrag. Er möchte „Klarheit“ (6) schaffen und zu einem „erhellenden Bild“ (10) der „Stadt als solcher“ beitragen. Ob dieses Bild tatsächlich allen Städten gerecht werden kann und wie es sich zu kritischen Perspektiven verhält, erfahren wir jedoch nicht. Nur sein Vergleich der Stadt mit einem „Werkzeug“ - dass sie ein „Tool“ (13) sei, das stets Antworten auf neue Probleme hervorbringe - deutet an, dass weniger kommensurable bzw. „zu handhabende“ (6) Merkmale diesem Wunschbild zum Opfer fallen könnten.

\section{Vexierbilder der Stadt und eine Definition}

Sein „neuer Weg“ (9) führt Prell, wie angedeutet, in zahlreiche Disziplinen, Theorieansätze und Sprachen, welche helfen sollen, „die Stadt“ zu entschlüsseln. Zu den Disziplinen, die sich mit Städten beschäftigen, zählt Prell Urbanistik und (Stadt-)Soziologie, Ökonomie, Geographie und Ökologie sowie Philosophie, Raum- und Stadtplanung, Rechts- und Politikwissenschaft, Architektur und die Geschichtswissenschaften. Seine Erkenntnisse zu deren verschiedenen „Stadtbegriffen“ präsentiert er zudem in einer umfangreichen Tabelle (39-42): Urbanistik und Philosophie besäßen das umfassendste Verständnis der Stadt, während die Geschichtswissenschaften nur „Einzelfälle“ interessierten. Die (Stadt-)Soziologie habe die „Deutungshoheit“, zeige aber das Manko, dass Stadt und Gesellschaft hier nicht ausreichend unterschieden werden (17 ff.). Das leiste erst die Politikwissenschaft (35), der Prell gar die Rolle einer „Königsdisziplin“ unter den Stadtwissenschaften zutraut (33 f.). Warum diese Leitfunktion benötigt wird, führt er aber nicht aus. Alle Disziplinen, so seine Hauptkritik, verfügten über keinen „scharfen Stadtbegriff“: Deshalb wüssten wir „[w]issenschaftlich gesehen letztlich nicht, was eine Stadt ist“ (37). Das aber führe dazu, dass einerseits diverse Stadtbegriffe nicht bewertet werden könnten und andererseits niemand einschätzen könne, was die heutige politische Bedeutung der Städte sei (38).

Dieser Befund führt Prell anschließend zu verschiedenen Stadttheorien (43). Warum er (nur) eine vormoderne Position referiert und ansonsten hauptsächlich Soziolog_innen berücksichtigt, belässt er aber im Dunkeln. Sicher wären andere Schlüsseltexte (vgl. Eckardt 2016) denkbar gewesen, die besser die Bandbreite der Forschung illustrieren. Der Werkzeug-Vergleich scheint noch am besten bei dem Philosophen Aristoteles zu funktionieren, demzufolge die Stadt dem Menschen helfe, „höhere Fähigkeiten“ zu entwickeln 
und „geistige Bedürfnisse“ zu befriedigen (Prell 2020: 43). Ähnlich scheint es bei Sombart, der urbane „Vielfalt“ eng mit ihrer „Multifunktionalität“ zusammen denkt. Prell kritisiert jedoch, dass hier die „Vielfalt“ schon zu groß werde und unklar bleibe, wie die vielfältigen „Puzzleteile zusammenpassen“ (44). Eindeutiger seien Weber, Simmel oder Louis Wirth (als Vertreter der Chicago School), die die Stadt als Einheit bzw. „Form“ (49) denken.

Wird der Soziologe Jürgen Friedrichs damit zitiert, dass er die Stadt ganz in Gesellschaft auflöse wolle (50), lobt Prell anschließend Saskia Sassens Konzept der Global City dafür, dass sie einen zwar eingeschränkten, aber funktionalen Begriff der Stadt als „Schauplatz globaler Prozesse“ (51) entwickle. Den Geographen Ash Amin und Stephen Graham gelinge es, im Begriff der Multiplex City Vielfalt und Einheit zusammenzudenken (55). Alle zusammen (inkl. des abschließend präsentierten Ansatzes der „offenen Stadt"von Richard Sennett; 57 f.) stimmten aber nur darin überein, Stadt als „Dichte und Vielfalt“ zu verstehen. Weitere Gemeinsamkeiten gebe es nicht. Prell reicht das nicht. Deshalb unternimmt er seinen letzten Versuch, zu einer „übergreifenden Definition“ zu gelangen, über die Sprache.

Die Anregung dazu fand er bei Sombart, allerdings dehnt Prell dessen Analyse auf zwölf Sprachen aus (ägyptische Hieroglyphen, Altgriechisch, Latein, Französisch, Englisch, Spanisch, Deutsch, Russisch, Arabisch, Hindi, Chinesisch, Japanisch; vgl. Prell 2017). Neben Lexika- und Wörterbücherrecherchen unternahm Prell sogar ethnografische Untersuchungen und fragte selbst bei „professionellen Dolmetscher[n] und Übersetzungsbüros“ nach (2016: 135 ff.). Letztlich besitze „die Stadt“ fünf Merkmale (2020: 77-80): Dichte, Vielfalt, Einheit und Struktur bildeten eine „Form“, die zugleich den Rahmen für das fünfte Merkmal bereitstelle: die „Kreativität“ des städtischen Handelns. Zusammengefasst begreift Prell die Stadt somit als „strukturierte, Vielfalt einschließende Einheit kreativer Verdichtung“ (80).

\section{Stadtthemen und Stadtkonzepte}

Diesen Begriff konfrontiert Prell anschließend, im vierten Kapitel, mit der „Praxis“. Darunter versteht er allerdings weder Methoden der Stadtforschung (vgl. Eckardt 2014) noch Verwaltungs- und kommunalpolitisches Handeln. „Praxis“ beschränkt sich hier allein auf „Stadtkonzepte“ und einige „Stadtthemen“ (Prell 2020: 120 ff.), in die der Autor einführt. Abermals lässt er im Dunkeln, wie diese Engführung rechtfertigbar ist. Im Sinne seines „holistischen Ansatzes“ wäre zu erwarten gewesen, dass ein solcher Schritt gut begründet wird, um sich gegen den Vorwurf des Reduktionismus zu schützen. Prell scheint es aber mehr darum zu gehen, die Wirksamkeit seines Begriffs zu demonstrieren.

Rund die Hälfte der Stadtkonzepte (Megacity, Global City, Hauptstadt, Arrival City, Smart City, Neoliberale Stadt, Virus City und Shrinking City) findet man auch im „Handbuch Stadtkonzepte“(Rink/Haase 2018), auf das Prell wiederholt verweist. Er versteht sie als Vermittler ,zwischen der Stadt als Ganzem" (Prell 2020: 83) und konkreten Herausforderungen. Alle führt Prell nach dem gleichen Schema ein: Zunächst präsentiert er Charakteristika und „Funktionen“ jedes Konzepts, im Anschluss wird nach Merkmalen des hintergründigen Stadtbegriffs gefragt und schließlich nach der „Antwort“, 
welche die Konzepte zu geben versuchen $(85,92)$. So schreibt er etwa zur Arrival City (96 ff.), diese sei auf Armutsviertel und informelle Siedlungen ausgerichtet, in denen sich viele „Einwanderer konzentrieren“. Das Konzept zeige die Chancen dieser Siedlungsformen, kritisiere aber auch eine fehlende offizielle Planung. Anders als im Fall der Global City (88 ff.) gehe es dabei nicht um Ranking oder eine Stadtvision. Vielmehr seien Arrival Cities die „Antwort“ auf „weltweite Wanderungsbewegungen“ (99). Ihr Stadtbegriff konzentriere sich aufVielfalt, Dichte, Einheit und Kreativität, während Strukturen kaum eine Rolle spielten.

Sind Konzepte wie die Megacity (84) oder die Hauptstadt (91) durchaus bekannt, integriert Prell mit der Virus City (108) ein neues Konzept, das Überlegungen zu den Folgen der Covid-19-Pandemie auf die Stadtplanung verkörpert. Im implizierten Stadtbegriff würden Eigenschaften wie Vielfalt, Dichte und Kreativität (110) an Bedeutung verlieren, sodass sich das Konzept als Antipode zur „offenen Stadt“ eigne.

Prell merkt an, dass sich die Konzepte meist auf nur „ein Thema“ konzentrierten und „die Reststadt“ darin wenig sichtbar sei. Zugleich legten etwa die Smart City oder die Neoliberale Stadt (99 ff.) wenig Wert auf „Verdichtung“ und „Vielfalt“ (118), während Megacity und Global City die „Einheit“ der Stadt vernachlässigten, was freilich nicht heiße, dass diese Merkmale auch „in der Realität nicht existieren“ (113). Am Ende bleibt allerdings unklar, was Prell mit diesen Erkenntnissen im Weiteren anfängt. Die sich andeutende kritische Perspektive auf „Stadtkonzepte“ (115 ff.) wird nicht weiterverfolgt.

Stattdessen beschäftigt sich Prell auf gerade einmal 15 Seiten mit verschiedenen „Stadtthemen“, die „derzeit besonders heftig diskutiert [werden]“(134). Unter „Zuwanderung und Abwanderung“, „Gesellschaft und Wirtschaft“ oder „Wohnen und Leben“ werden dabei ganze Themenbereiche zusammengefasst. Der Fokus liegt erneut auf westlichen Städten, während Inhalte und Debatten allenfalls schlaglichtartig eingeführt werden. Zielkonflikte wie jener zwischen automobilem Individualverkehr und dem Bedarf nach Klimaschutz und Ressourcenschonung (125 f.) - die Prell unter der Überschrift „Bewegung und Stillstand“ abhandelt - sind jedoch längst globale Angelegenheiten. Und das betrifft auch Spaltungen, die auf Strukturreformen („Digitalisierung“) oder Auswirkungen demografischer Prozesse zurückgehen (und unter „analog und digital“ bzw. „Stadt und Land“ vom Autor angedeutet werden). „Stadt und Umwelt“ beleuchtet sehr kurz die ambivalente Rolle der (Groß-) Städte in Zeiten des Klimawandels (133). Mit Rückblick auf die Single-issueOrientierung der Stadtkonzepte hebt Prell am Schluss nochmals hervor, dass es wichtig sei, die „Wechselwirkungen“ zwischen allen städtischen Herausforderungen im Auge zu behalten. Auch das fünfte Kapitel stellt diese Anforderung ins Zentrum. Prell hebt einmal mehr hervor, dass nur mit einem „Gesamtbild“ der Stadt (137) - des „,besten Werkzeugs“ (137) - zukünftige städtische Probleme gemeistert werden könnten.

\section{Die Stadt - gegen den Strich gelesen (Fazit)}

Obwohl von geringem Seitenumfang, bietet Die Stadt diverse Einblicke des Autors zur heutigen Stadtforschung. Dennoch beschränkt sich der „Überblick“ in den meisten Fällen auf Inhalte, die er zur Rechtfertigung seines 
„holistischen Ansatzes“ und zur Verteidigung eines allgemeingültigen Stadtbegriffes nutzen kann. Diese nicht unproblematische Selektivität wird bei den Autor_innen und Themen deutlich, aber auch beim Praxisverständnis - und noch am Stadtbegriff selbst. Für eine „Einführung für die Sozialwissenschaften" ist sicher ungünstig, dass er sowohl die sozialwissenschaftlichen Methoden ausspart als auch Themen wie Urbanität, das Verhältnis von Öffentlichkeit und Privatheit, die Ungleichheitsforschung oder auch neuere (z. B. feministische und postkoloniale) Ansätze (Eckardt 2014; Häußermann/Siebel 2004; Varma 2012). Auffällig wenig liest man über die Diversität, Dynamik und symbolische Bedeutung städtischer Räume oder über Konflikte um deren (Re-)Produktion und Aneignung (Löw 2018; Lefebvre 1991). Dabei ist gerade die Stadtplanung, in der jene „Stadtkonzepte“ in die Praxis übersetzt werden, immer wieder mit Kämpfen um die Funktion und Bedeutung städtischer Räume konfrontiert - nicht nur im globalen Süden, auf den der Autor ebenfalls kaum eingeht (Prell 2020: 138).

Der achtenswerte Versuch eines Gesamtüberblicks zu allen städtischen Disziplinen (Psychologie, Kriminalistik oder die Literaturwissenschaften könnten ergänzt werden) leidet an deren schlaglichtartiger Darstellung, die kaum in Fachdebatten einsteigt (mit Ausnahme von Soziologie und Politikwissenschaft). Man muss das als vertane Chance werten, schließlich wäre deren genauere Präsentation enorm nützlich für eine Einführung. Stattdessen bietet Prell eine eigenwillige Interpretation von „Interdisziplinarität“, die fordert, sich „mit allen [Stadt-]Disziplinen“ (10, 36; Hervorhebung d. A.) zu beschäftigen. Anders als in dieser kaum einlösbaren Forderung wird unter Interdisziplinarität im Allgemeinen die Zusammenarbeit verschiedener Disziplinen verstanden, deren Kooperation diverse Perspektiven und Methoden zu gemeinsamen Ergebnissen zusammenführt - ohne dass übrigens dabei eine Disziplin die Leitfunktion übernehmen müsste. Der Nutzen, den Prell für das „Gesamtbild“ und die „übergreifende Definition“ (37, 137) verspricht, bleibt letztlich vage und ebenso zweifelhaft wie der Werkzeug-Vergleich. Überhaupt wirken manche der Metaphern durchaus verräterisch.

So vergleicht Prell die Stadt auch mit einem „Puzzle“ (36), in das sich jede Einzeldisziplin wie ein „schön gearbeiteter Mosaikstein“ einfügen soll (10), und jene fünf Merkmale bezeichnet er gar als „Gene der Stadt“ (77). Diese Vergleiche legen nahe, dass es schon ein Bild oder einen Bauplan „der Stadt“ gebe, die nur entdeckt und dechiffriert zu werden bräuchten. Wie bei der Werkzeug-Metapher schwingt dabei mit, dass es auch „falsche“ Verwendungen geben kann, die das „Ganze“ gefährden könnten. Ähnlich vorbelastet erscheint sein Anspruch auf „Klarheit“ und „Aufklärung“, wenn darin Wissen mit „Macht“ gleichgesetzt wird (6) und es im Anschluss heißt, dass die „Eigenschaften eines Werkzeugs so genau wie möglich zu kennen“ dabei helfe, es „zu handhaben“ (6). Doch kann man Städte überhaupt wie Werkzeuge „handhaben“? Wie weit trägt dieser Vergleich wirklich?

Prell, so scheint es, hebt damit vor allem auf historische Funktionen der Stadt ab, die unsere Handlungs- und Entwicklungsmöglichkeiten in Städten vermehrt haben. Freilich gingen sie aus einer Konzentration und Verdichtung hervor, die nicht nur Vorteile und auch nicht allein neue „Funktionen“ brachten. Denn Städte sind nicht nur technisch-materielle, sondern ebenso kulturelle und symbolische Artefakte, in denen sich Räume 
sowie soziale Praktiken überlagern und zudem häufig zu Konflikten führen. Eine funktionalistische Interpretation läuft dagegen Gefahr, gerade das Unstimmige und Überraschende, ja das Ambivalente (Krusche 2017) und Redundante (Kling 2020), kurz: das (scheinbar) Unnütze an Städten zu übersehen.

In ähnlicher Weise kann auch die Definition einer „abstrahierten Stadt“ (Eckardt 2016: 2) bei Prell kaum überzeugen. Das liegt schon daran, dass Eigenschaften wie Einheit, Dichte, Struktur oder Vielfalt selbst kontextabhängig sind und entsprechend zu historisieren wären. Dabei handelt es sich um einen der Hauptpunkte jener anhaltenden Soziologiedebatte über die Eigenständigkeit der Städte (Häußermann/Siebel 2004; Lefebvre 2014; Saunders 1987). Überdies hat schon Soja (2000: $145 \mathrm{ff}$.) gezeigt, dass längst in manchen Diskursen selbst städtische Merkmale wie Dichte („Exopolis“; 233 ff.), Einheit („fractal city“; 264 ff.) oder Vielfalt („Carceral Archipelago“; 298 ff.) infrage gestellt werden.

Behält man diese Schwächen im Auge, fördert zumindest Prells Prüfung der Stadtkonzepte einige interessante Resultate zutage. Zu Recht betont er im vierten Kapitel, dass besagte Konzepte die Stadt häufig auf einen bestimmten Aspekt reduzierten. Das führe dazu, dass in der Stadtplanung zwischen ihnen eine Konkurrenz um „Ressourcen“ und „Deutungshoheit“ (Prell 2020: 119) entstehe. Gerade die „wirkmächtigsten Konzepte“ zeigten „fundamentale Schwächen“ (107), etwa weil sie die „Einheit“ der Stadt - im Sinne aller Bewohner_inneninteressen - ignorierten (118). Die Planung von Smart Cities setze „stark oder sogar ausschließlich“ auf Digitalisierung und drohe über diese monothematische Orientierung den fehlenden Zugang mancher Menschen zu technischer Ausstattung oder Bildung zu übersehen (102). Ähnlich einseitig wirke die Planung von Global Cities, wenn es dabei nur um internationales Ansehen und die Einbettung in den globalen Wirtschaftsraum gehe. Soziale Folgen wie die „Aufspaltung“ der Bevölkerung gerieten dabei aus dem Blick (90). Die neoliberale Entwicklung könne zwar den Wettbewerb steigern, aber nicht selbst verhindern, dass die „Ökonomisierung“ dysfunktionale Folgen zeitige (106 f.). „Je schärfer“ die Konzepte auf nur ein Merkmal abheben, so fasst es Prell zusammen, „desto stärker [blenden sie] die nicht betroffenen Teile der Stadt" aus (115). Es sind Erkenntnisse wie diese, die ein kritisches Potenzial andeuten, das der Band allerdings zu wenig ausgeschöpft hat.

\section{Autor_innen}

Alexander Krahmer ist Stadtsoziologe und beschäftigt sich aktuell mit kritischer Stadtforschung, Konflikttheorien sowie Gerechtigkeits- und Sicherheitsforschung im urbanen Kontext. alexander.krahmer@ufz.de 


\section{Literatur}

Eckardt, Frank (2014): Stadtforschung. Gegenstand und Methoden. Wiesbaden: Springer VS. Eckardt, Frank (Hg.) (2016): Schlüsselwerke der Stadtforschung. Wiesbaden: Springer VS.

Häußermann, Hartmut / Siebel, Walter (2004): Stadtsoziologie. Eine Einführung. Frankfurt am Main/New York: Campus.

Kling, Norbert (2020): The redundant city. A multi-site enquiry into urban narratives of conflict and change. Bielefeld: transcript.

Krusche, Jürgen (Hg.) (2017): Die ambivalente Stadt. Gegenwart und Zukunft des öffentlichen Raums. Berlin: JOVIS.

Lefebvre, Henri (1991): The production of space. Oxford: Blackwell.

Lefebvre, Henri (2014): Die Revolution der Städte. La révolution urbaine. Neuausgabe mit einer Einführung von Klaus Ronneberger. Hamburg: CEP Europäische Verlagsanstalt.

Löw, Martina (2018): Vom Raum aus die Stadt denken. Grundlagen einer raumtheoretischen Stadtsoziologie. Bielefeld: transcript.

Prell, Uwe (2005): Berlin - Bühne des Wandels. Ein Reisebericht. Berlin: Berliner Wissenschafts-Verlag.

Prell, Uwe (2016): Theorie der Stadt in der Moderne. Kreative Verdichtung. Opladen u. a.: Barbara Budrich.

Prell, Uwe (2017): Die Stadt: zwölf Sprachen - fünf Bedeutungen. Ein Beitrag zur Theorie der Stadt. Opladen u. a.: Barbara Budrich.

Prell, Uwe (2020): Die Stadt. Eine Einführung für die Sozialwissenschaften. Opladen u. a.: Barbara Budrich.

Prell, Uwe / Ulrich, Horst (1992): Berlin Handbuch. Das Lexikon der Bundeshauptstadt. Berlin: FAB Verlag.

Rink, Dieter / Haase, Annegret (Hg.) (2018): Handbuch Stadtkonzepte. Analysen, Diagnosen, Kritiken und Visionen. Opladen u. a.: Barbara Budrich.

Saunders, Peter (1987): Soziologie der Stadt. Frankfurt am Main/New York: Campus.

Soja, Edward W. (2000): Postmetropolis. Critical studies of cities and regions. Oxford: Blackwell.

Varma, Rashmi (2012): The postcolonial city and its subjects: London, Nairobi, Bombay. New York u. a.: Routledge. 